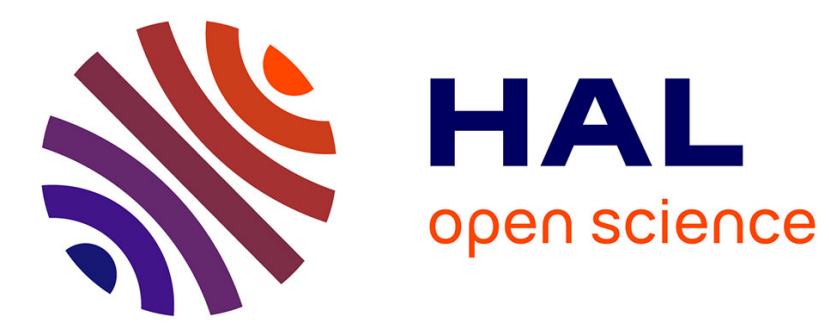

\title{
Faster Chosen-Key Distinguishers on Reduced-Round AES
}

Patrick Derbez, Pierre-Alain Fouque, Jérémy Jean

\section{To cite this version:}

Patrick Derbez, Pierre-Alain Fouque, Jérémy Jean. Faster Chosen-Key Distinguishers on ReducedRound AES. Progress in Cryptology - INDOCRYPT 2012, Dec 2012, Kolkata, India. pp.19, 10.1007/978-3-642-34931-7_14. hal-01094335

\section{HAL Id: hal-01094335 \\ https://hal.inria.fr/hal-01094335}

Submitted on 12 Dec 2014

HAL is a multi-disciplinary open access archive for the deposit and dissemination of scientific research documents, whether they are published or not. The documents may come from teaching and research institutions in France or abroad, or from public or private research centers.
L'archive ouverte pluridisciplinaire HAL, est destinée au dépôt et à la diffusion de documents scientifiques de niveau recherche, publiés ou non, émanant des établissements d'enseignement et de recherche français ou étrangers, des laboratoires publics ou privés. 


\title{
Faster Chosen-Key Distinguishers on Reduced-Round AES
}

\author{
Patrick Derbez, Pierre-Alain Fouque, and Jérémy Jean \\ École Normale Supérieure, 45 Rue d'Ulm, 75005 Paris, France \\ \{Patrick.Derbez, Pierre-Alain.Fouque, Jeremy.Jean\} @ens.fr
}

\begin{abstract}
In this paper, we study the AES block cipher in the chosen-key setting. The adversary's goal of this security model is to find triplets $\left(m, m^{\prime}, k\right)$ satisfying some properties more efficiently for the AES scheme than generic attacks. It is a restriction of the classical chosen-key model, since as it has been defined originally, differences in the keys are possible. This model is related to the known-key setting, where the adversary receives a key $k$, and tries to find a pair of messages $\left(m, m^{\prime}\right)$ that has some property more efficiently than generic attacks. Both models have been called open-key model in the literature and are interesting for the security of AES-based hash functions.

Here, we show that in the chosen-key setting, attacking seven rounds (resp. eight rounds) of $\mathrm{AES}-128$ can be done in time and memory $2^{8}$ (resp. $2^{24}$ ) while the generic attack would require $2^{64}$ computations as a variant of the birthday paradox can be used to predict the generic complexity. We have checked our results experimentally and we extend them to distinguisers of AES-256.
\end{abstract}

Keywords: AES, Open-key Model, Chosen-key Distinguisher, Practical Complexities.

\section{Introduction}

The Advanced Encryption Standard (AES) [16] is nowadays the subject of many attention since attacks coming from hash function cryptanalysis have put its security into question. Related-key attacks and meet-in-the-middle attacks that begin in the middle of the cipher (also known as splice-and-cut attacks) have been proposed to attack the full number of rounds for each AES versions [1,2,4], while other techniques exist for smaller version [5]. This interesting connection between hash functions and block ciphers shows that any improvement on hash function cryptanalysis can be useful for attacking block ciphers and vice-versa.

In this work, we study another model that has been suggested to study the security of hash functions based on AES components. Knudsen and Rijmen [9] have proposed to consider known-key attacks since in the hash function domain, the key is usually known and the goal is to find two input messages that satisfy some interesting relations. In some setting, a part of the key can also be chosen (for instance when salt is added to the hash function) and therefore, cryptanalysts have also consider the model where the key is under the control of the adversary. The latter model has been called chosen-key model and both models belong to the open-key model. The chosen-key model has been popularized by Biryukov et al. in [2], since a distinguisher in this model has been extended to a related-key attack on the full AES -256 version.

Related Work. Knudsen and Rijmen in [9] have been the firsts to consider known-key distinguishers on AES and Feistel schemes. The main motivations for this model are the following:

- if there is no distinguisher when the key is known, then there will also be no distinguisher when the key is secret, 
- if it is possible to find an efficient distinguisher, finding partial collision on the output of the cipher more efficiently than birthday paradox would predict even though the key is known, then the authors would not recommend the use of such cipher,

- finally, such model where the key is known or chosen can be interesting to study the use of cipher in a compression function for a hash function.

In the same work, they present some results on Feistel schemes and on the AES. Following this work, Minier et al. in [14] extend the results on AES on the Rijndael scheme with larger block-size.

In [2], Biryukov et al. have been the firsts to consider the chosen-key distinguisher for the full 256-bit key AES. They show that in time $q \cdot 2^{67}$, it is possible to construct $q$ multicollision on Davies-Meyer compression function using AES-256, whereas for an ideal cipher, it would require on average $q \cdot 2^{\frac{q-1}{q+1} 128}$ time complexity. In these chosen-key distinguishers, the adversary is allowed to put difference also in the key. Later, Nikolic et al. in [15], describe known-key and chosen-key distinguishers on Feistel and Substitution-Permutation Networks (SPN). The notion of chosen-key distinguisher is more general than the model that we use: here, we let the adversary choose the key, but it has to be the same for the input and output relations we are looking for. We do not consider related-keys in this article. Then in [12], rebound attacks have been used to improve known-key distinguishers on AES by Mendel et al. and in [8], Gilbert and Peyrin have used both the SuperSBox and the rebound techniques to get a known-key distinguisher on 8-round AES-128. Last year at FSE, Sasaki and Yasuda show in [18] an attack on 11 Feistel rounds and collision attacks in hashing mode also using rebound techniques, and more recently, Sasaki et al. studied the known-key scenario for Feistel ciphers like Camellia in [17].

Our Results. In this paper, we study 128- and 256-bit reduced versions of AES in the (single) chosen-key model where the attacker is challenged to find a key $k$ and a pair of messages $\left(m, m^{\prime}\right)$ such that $m \oplus m^{\prime} \in E$ and $\operatorname{AES}_{k}(m) \oplus \operatorname{AES}_{k}\left(m^{\prime}\right) \in F$, where $E$ and $F$ are two known subspaces. On AES-128, we describe in that model a way to distinguish the 7-round AES in time $2^{8}$ and the 8 -round AES in time $2^{24}$. In the case of the 7 -round distinguisher, our technique improves the $2^{16}$ time complexity of a regular rebound technique [13] on the SubBytes layer by computing intersections of small lists. The 8-round distinguisher introduces a problem related the SuperSBox construction where the key parameter is under the control of the adversary. As for AES-256, the distinguishers are the natural extensions of the ones on AES-128. Our results are reported in Table 1. We have experimentally checked our results and examples are provided in the appendices. We believe our practical distinguishers can be useful to construct non-trivial inputs for the AES block cipher to be able to check the validity of some theoretical attacks, for instance [7].

Outline of the paper. The paper is organized as follows. We begin in Section 2 by recalling the AES and the concept of SuperSBox. Then in Section 3.1, we precise the chosen-key model in the ideal case to be able to compare our distinguishers to the ideal scenario. Section 3.1 describes the main results of the AES-128 and Section 4 shows how to apply similar results to the AES-256.

\section{Description of the AES}

The Advanced Encryption Standard [16] is a Substitution-Permutation Network that can be instantiated using three different key bit-lengths: 128, 192, and 256. The 128-bit plaintext 
Table 1: Comparison of our results to previous ones on reduced-round distinguishers of the AES-128 in the open-key model. Results from [1] are not mentioned since we do not consider related-keys in this paper.

\begin{tabular}{ccccccc}
\hline Target & Model & Rounds & Time & Memory & Ideal & Reference \\
\hline & Known-key & 7 & $2^{56}$ & - & $2^{58 \star}$ & {$[9]$} \\
Known-key & 7 & $2^{24}$ & $2^{16}$ & $2^{64}$ & {$[12]$} \\
& Single-chosen-key & 7 & $2^{22}$ & - & $2^{64}$ & {$[3]$} \\
& Single-chosen-key & $\mathbf{7}$ & $\mathbf{2}^{8}$ & $\mathbf{2}^{8}$ & $\mathbf{2}^{64}$ & Section 3.2 \\
& Known-key & 8 & $2^{48}$ & $2^{32}$ & $2^{64}$ & {$[8]$} \\
& Single-chosen-key & 8 & $2^{44}$ & - & $2^{64}$ & {$[3]$} \\
& Single-chosen-key & $\mathbf{8}$ & $\mathbf{2}^{24}$ & $\mathbf{2}^{16}$ & $\mathbf{2}^{64}$ & Section 3.3 \\
\hline \multirow{2}{*}{ AES-256 6 Single-chosen-key } & $\mathbf{7}$ & $\mathbf{2}^{8}$ & $\mathbf{2}^{8}$ & $\mathbf{2}^{64}$ & Section 4.1 \\
& Single-chosen-key & $\mathbf{8}$ & $\mathbf{2}^{8}$ & $\mathbf{2}^{8}$ & $\mathbf{2}^{64}$ & Section 4.2 \\
& Single-chosen-key & $\mathbf{9}$ & $\mathbf{2}^{24}$ & $\mathbf{2}^{16}$ & $\mathbf{2}^{64}$ & Section 4.3 \\
\hline
\end{tabular}

* Claimed by the authors as a very inaccurate estimation of the [ideal] complexity.

initializes the internal state viewed as a $4 \times 4$ matrix of bytes as values in the finite field $G F\left(2^{8}\right)$, which is defined via the irreducible polynomial $x^{8}+x^{4}+x^{3}+x+1$ over $G F(2)$. Depending on the version of the AES, $N_{r}$ rounds are applied to that state: $N_{r}=10$ for AES-128, $N_{r}=12$ for AES-192 and $N_{r}=14$ for AES-256. Each of the $N_{r}$ AES round (Figure 1) applies four operations to the state matrix (except the last one where we omit the MixColumns):

- AddRoundKey (AK) adds a 128-bit subkey to the state.

- SubBytes (SB) applies the same 8-bit to 8-bit invertible S-Box S 16 times in parallel on each byte of the state,

- ShiftRows (SR) shifts the $i$-th row left by $i$ positions,

- MixColumns (MC) replaces each of the four column $C$ of the state by $M \times C$ where $M$ is a constant $4 \times 4$ maximum distance separable circulant matrix over the field $G F\left(2^{8}\right)$, $M=\operatorname{circ}(2,3,1,1)$.

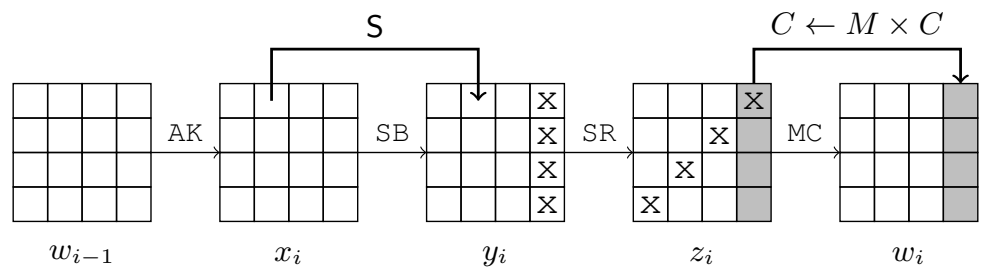

Figure 1: An AES round applies $\mathrm{MC} \circ \mathrm{SR} \circ \mathrm{SB} \circ \mathrm{AK}$ to the state. There are $N_{r}=10$ rounds in $\mathrm{AES}-128$.

After the $N_{r}$-th rounds has been applied, a final subkey is added to the internal state to produce the ciphertext. The key expansion algorithm to produce the $N_{r}+1$ subkeys for AES-128 is described in Figure 2(a), and in Figure 2(b) for the AES-256. We refer to the official specification document [16] for further details.

SuperSBox. In [6], Rijmen and Daemen introduced the concept of SuperSBox to study two rounds of AES. This transformation sees the composition $S B \circ A K(k) \circ M C \circ S B$ as four parallel applications of a 32-bit S-Box, and has been useful for several cryptanalysis works, see for instance $[8,10]$. Abusing notations, in the sequel, we call SuperSBox keyed by the key $k$ the transformation that applies this composition to a single AES-column. In that 


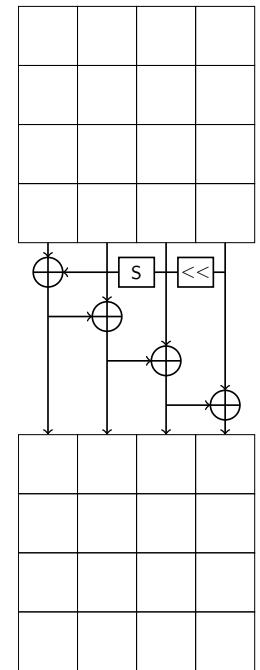

(a) AES-128.

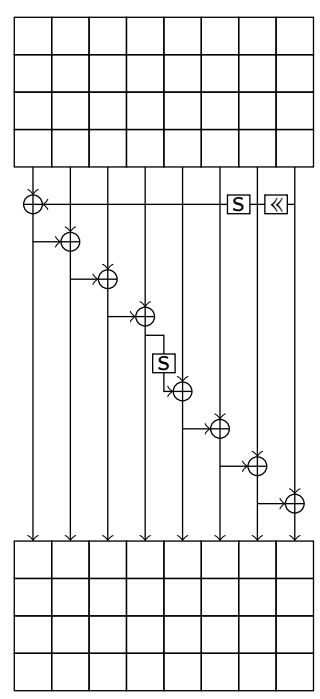

(b) AES-256.

\begin{tabular}{|c|c|c|c|}
\hline 0 & 4 & 8 & 12 \\
\hline 1 & 5 & 9 & 13 \\
\hline 2 & 6 & 10 & 14 \\
\hline 3 & 7 & 11 & 15 \\
\hline
\end{tabular}

(c) Naming.

Figure 2: Key schedules of the variants of the AES (AES-128 and AES-256) - Naming of bytes in a state.

context, the key $k$ which parameterized the SuperSBox is also a 32-bit AES-column. We denote that operation by SuperSBox $_{k}$.

Notations. In this paper, we count the AES rounds from 0 and we refer to a particular byte of an internal state $x$ by $x[i]$, as depicted in Figure 2(c). Moreover, as shown in Figure 1, in the $i$ th round, we denote the internal state after AddRoundKey by $x_{i}$, after SubBytes by $y_{i}$, after ShiftRows by $z_{i}$ and after MixColumns by $w_{i}$. To refer to the difference in a state $x$, we use the notation $\Delta x$.

\section{Chosen-key distinguishers}

\subsection{Limited Birthday Distinguishers}

In this section, we precise the distinguishers we are using. Our first goal is to distinguish the AES-128 from an ideal keyed-permutation in the chosen-key model. We will derive distinguishers for AES-256 afterwards. We are interested in the kind of distinguishers where the attacker is asked to find a key and a pair of plaintext whose difference is constrained in a predefined input subspace such that the ciphertext difference lies in an other predefined subspace.

Property 1 Given two subspaces $E_{\text {in }}$ and $E_{\text {out }}$, a key $k$ and a pair of messages $(x, y)$ verify the property on a permutation $P$ if $x+y \in E_{\text {in }}$ and $P(x)+P(y) \in E_{\text {out }}$.

This type of distinguisher looks like the limited birthday distinguishers introduced by Gilbert and Peyrin in [8] with a very close lower bound proved in [15], except that we allow the attacker more freedom; namely, in the choice of the key bits. To determine how hard this problem is, we need to compare the real-world case to the ideal scenario. In the latter, the attacker faces a family ${ }^{1}$ of pseudo-random permutations $\mathcal{F}: \mathcal{K} \times \mathcal{D} \longrightarrow \mathcal{D}$, and would run a limited birthday distinguisher on a particular random permutation $F_{k}$ to find a pair of

\footnotetext{
${ }^{1}$ where both $\mathcal{K}$ and $\mathcal{D}$ are $\{0,1\}^{128}$ in the case of AES-128.
} 
messages that conforms to the subspace restrictions of Property 1. The additional freedom of this setting does not help the attacker to find the actual pair of messages that verifies the required property, because the permutation $F_{k}$ has to be chosen beforehand. Put it another way, the birthday paradox is as constrained as if the key were known since no difference can be introduced in the key bits.

Therefore, even if we let the key to be chosen by the attacker, the limited birthday distinguisher from [8] applies in the same way. For known $E_{\text {in }}$ and $E_{\text {out }}$, we denote $n_{i}=$ $\operatorname{dim}\left(E_{\text {in }}\right)$ and $n_{o}=\operatorname{dim}\left(E_{\text {out }}\right)$. In terms of truncated differences, $n_{i}$ (resp. $n_{o}$ ) represents the number of independent active truncated differences in the input (resp. output) of a random permutation $F_{k} \in \mathcal{F}$ (see Figure 3). Both $n_{i}$ and $n_{o}$ range in the interval between 0 and $n$, where $n=16$ in the case of AES. Without loss of generality, we assume that $n_{i} \leq n_{o}$ : the attacker thus considers $F_{k}$ rather than its inverse, as it is easier to collide on $n-n_{o}$ differences than on $n-n_{i}$. The attacker continues by constructing two lists $L$ and $L^{\prime}$ of

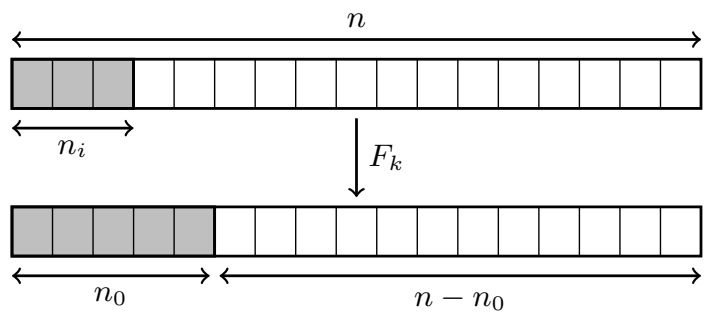

Figure 3: Assuming $n_{i} \leq n_{o}$, the attacker searches for a pair of input to the random permutation $F_{k}$ differing in $n_{i}$ known byte positions such that the output differs in $n_{o}$ known byte positions. A gray cell indicates a byte with a truncated difference.

$2^{8 n_{i}}$ plaintexts each by choosing a random value for the $n-n_{i}$ inactive bytes of the input and considering all the $n_{i}$ active ones in $E_{i n}$. With a birthday paradox on the two lists $L$ and $L^{\prime}$, she expects a collision on at most $2 n_{i}$ bytes of the ciphertexts. In the event that $n-n_{o} \geq 2 n_{i}$, then $n-2 n_{i}$ bytes have not a zero-difference in the ciphertext. Hence, we need to restart the birthday paradox process about $2^{8\left(n-n_{o}-2 n_{i}\right)}$ times, which $\operatorname{costs} 2^{8\left(n-n_{o}-n_{i}\right)}$ in total. Otherwise, if $n-n_{o}<2 n_{i}$, then a single birthday paradox with lists of size $2^{8\left(n-n_{o}\right) / 2}$ is sufficient to get a collision on the $n-n_{o}$ required bytes in time $2^{8\left(n-n_{o}\right) / 2}$.

\subsection{Distinguisher for 7-round $A E S-128$}

We consider the 7-round truncated differential characteristic of Figure 4, where the differences in both the plaintext and the ciphertext lie in subspaces of dimension four. Indeed, the output difference lies in a subspace of dimension four since all the operations after the last SubBytes layer are linear. With respect to the description of the distinguisher (Section 3.1), the time complexity to find a pair of messages that conforms to those patterns in a family of pseudo-random permutations is $2^{64}$ basic operations.

The following of this section describes a way to build a key and a pair of messages that conform to the restrictions in time $2^{8}$ basic operations using a memory complexity of $2^{8}$ bytes. This complexity has to be compared to $2^{16}$ operations, which is the time complexity expected for a straightforward application of the rebound attack [13] on the SubBytes layer of the AES. In that case, there are 16 random differential transitions around the 


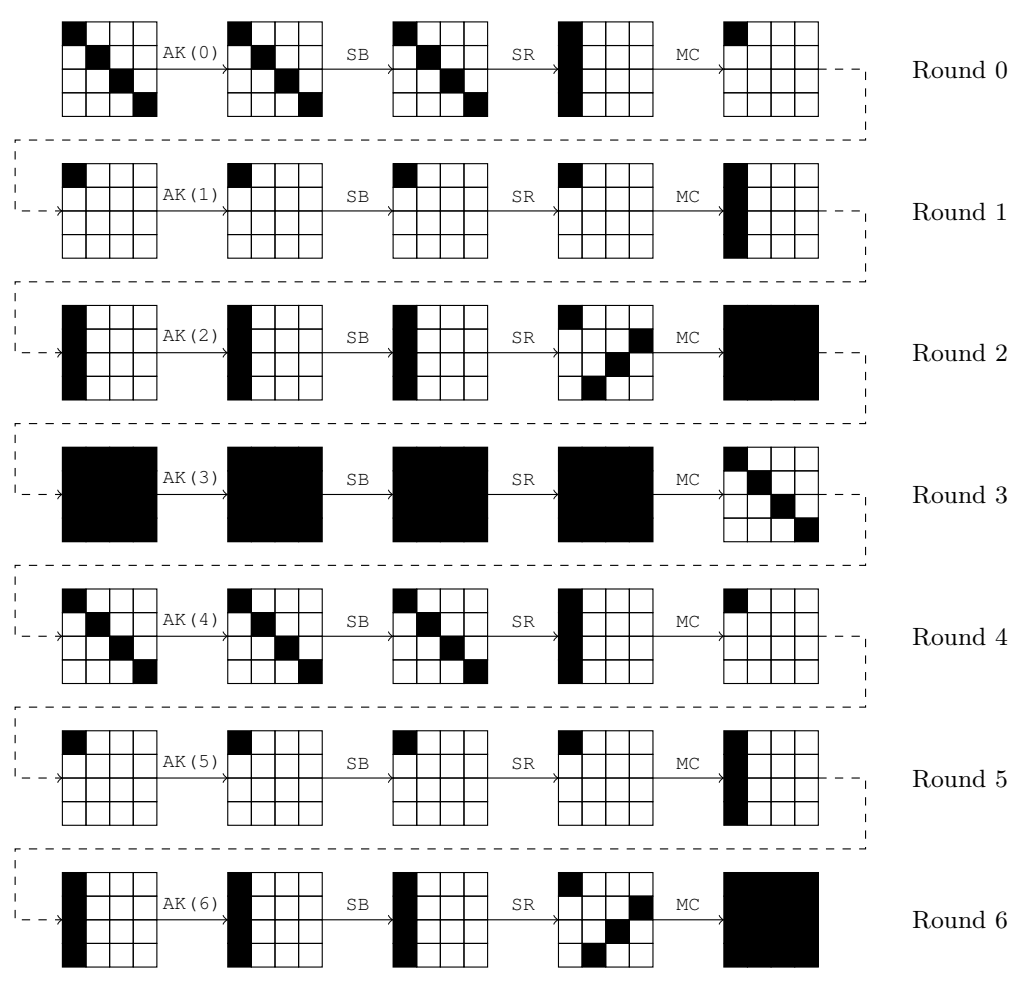

Figure 4: The 7-round truncated differential characteristic used to distinguish the AES-128 from a random permutation. Black bytes are active, white bytes are not.

AES S-Box, which happens to be all compatible ${ }^{2}$ with probability $2^{-16}$. Repeating with random differences $2^{16}$ times, we expect to find a pair of internal states that conforms to the randomized differences. In the following, we proceed slightly differently to reach a solution in time $2^{8}$.

In terms of freedom degrees, we begin by estimating the number of solutions that we expect to verify the truncated differential characteristic. There are 16 bytes in the first message, 4 more independent ones in the second message and 16 others in the key: that makes 36 freedom degrees at the input. On a random input, the probability that the truncated differential characteristic being followed depends on the amount of freedom degrees that we loose in probabilistic transitions within the MixColumns transitions: 3 in round 0 to pass one $4 \rightarrow 1$ truncated transition, 12 in round 3 to pass four $4 \rightarrow 1$ transitions and 3 again in round 4 for the last $4 \rightarrow 1$ transition. In total, we thus expect

$$
2^{8 \times(16+4+16)} 2^{-8 \times(3+12+3)}=2^{8 \times 18}
$$

triplets $\left(m, m^{\prime}, k\right)$ composed by a pair $\left(m, m^{\prime}\right)$ of messages and a key $k$ to conform to the truncated differential characteristic of Figure 4. Hence, we have 18 freedom degrees left to find such a triplet.

First, we observe that whenever we find such a solution for the middle rounds (round 1 to round 4), we are ensured that all the rounds will be covered as in the whole truncated differential characteristic due to an outward propagation occurring with probability 1 . Hence, our strategy focuses on those rounds. The context is similar to the rebound scenario, where we first solve the inbound phase and then propagate it into the outbound phase.

\footnotetext{
${ }^{2}$ By compatible, we mean that we can find at least a pair of values that conforms to the differential transition. In the case of the AES S-Box, for a random differential transition $\delta \rightarrow \delta^{\prime}$, this is known to be possible with probability close to $1 / 2$.
} 


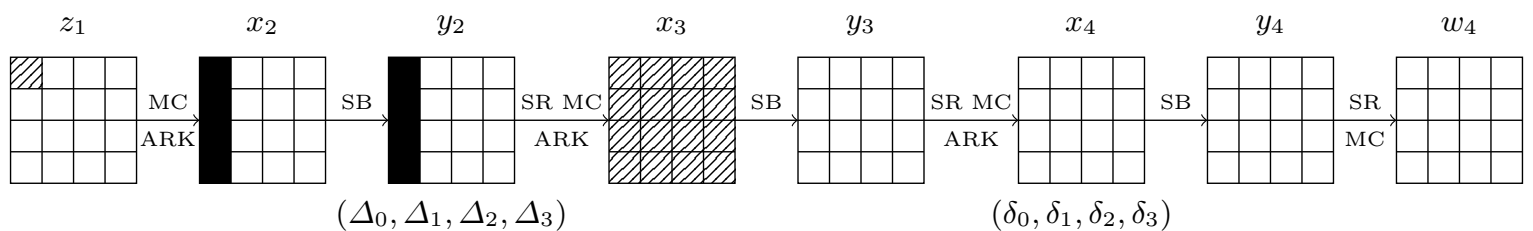

Figure 5: The 7-round distinguishing attack focuses of the middle rounds. Black bytes have known values and differences, gray bytes have known values, hatched bytes have known differences and white bytes have unknown values and/or differences.

To reduce the number of valid solutions, we begin by fixing some bytes (Figure 5) to a random value: $\Delta z_{1}$ and $x_{2}[0 . .3]$. Therefore, we can deduce the values and differences in the first column of $x_{2}$ and $y_{2}$, as well as the difference $\Delta x_{3}$ by linearity. Let $\left[\Delta_{0}, \Delta_{1}, \Delta_{2}, \Delta_{3}\right]^{\mathrm{T}}$ be the column-vector of deduced differences in $\Delta y_{2}$ and $\operatorname{diag}\left(\delta_{0}, \delta_{1}, \delta_{2}, \delta_{3}\right)$ the differences in the diagonal of $\Delta x_{4}$. Linearly, we can express the differences around the SubBytes layer of round 3 (see Figure 6). As a consequence, from the differential properties of the AES S-Box,

\begin{tabular}{|c|c|c|c|c|c|c|c|c|}
\hline \multicolumn{4}{|c|}{$\Delta x_{3}$} & & \multicolumn{4}{|c|}{$\Delta y_{3}$} \\
\hline $2 \Delta_{0}$ & $\Delta_{3}$ & $\Delta_{2}$ & $3 \Delta_{1}$ & & $14 \delta_{0}$ & $11 \delta_{1}$ & $13 \delta_{2}$ & $9 \delta_{3}$ \\
\hline$\Delta_{0}$ & $\Delta_{3}$ & $3 \Delta_{2}$ & $2 \Delta_{1}$ & SB & $13 \delta_{3}$ & $9 \delta_{0}$ & $14 \delta_{1}$ & $11 \delta_{2}$ \\
\hline$\Delta_{0}$ & $3 \Delta_{3}$ & $2 \Delta_{2}$ & $\Delta_{1}$ & & $14 \delta_{2}$ & $11 \delta_{3}$ & $14 \delta_{0}$ & $9 \delta_{1}$ \\
\hline $3 \Delta_{0}$ & $2 \Delta_{3}$ & $\Delta_{2}$ & $\Delta_{1}$ & & $13 \delta_{1}$ & $9 \delta_{2}$ & $14 \delta_{3}$ & $11 \delta_{0}$ \\
\hline
\end{tabular}

Figure 6: Differences around the SubBytes layer of round 3: each $\Delta_{j}$ is fixed, whereas the $\delta_{i}$ are yet to be determined.

for $i, j \in\{0, \ldots, 3\}, \Delta_{j}$ suggests $2^{7}$ different values for $\delta_{i}$ : we store them in the list $L_{i, j}$.

$$
L_{i, j}=\left\{\delta_{i} / \Delta_{j} \rightarrow \delta_{i} \text { is possible }\right\}
$$

Once done, we build the list $L_{i}$, for $i \in\{0, \ldots, 3\}$ :

$$
L_{i}=\bigcap_{j=0}^{3} L_{i, j}=\left\{\delta_{i} / \forall j \in\{0, \ldots, 3\}, \Delta_{j} \rightarrow \delta_{i} \text { is possible }\right\} .
$$

Each $L_{i, j}$ being of size $2^{7}$, we expect each $L_{i}$ to contain $2^{4}$ elements.

We continue by setting $\Delta x_{4}[0]$ to random value in $L_{0}$ and $x_{4}[0]$ to a random value, which allow to determine the value and difference in $y_{4}[0]$. Since the difference $\Delta y_{4}$ can only take $2^{8}$ values due to the MixColumns transition of round 4 , we also deduce $\Delta w_{4}$ and the remaining differences in $\Delta y_{4}$. The knowledge of $\Delta y_{4}$ suggests $2^{7}$ possible values for $\delta_{i}$. As before, we store them in lists called $T_{i}$, and we select a value for $\delta_{i}$ in $L_{i} \cap T_{i}$ (Figure 7). We expect each intersection to contain about $2^{3}$ elements. More rigorously, if we assume that the lists $L_{i, j}$ and $T_{i}$ are uniformly distributed, then the probability that $L_{0}, L_{1} \cap T_{1}, L_{2} \cap T_{2}$ and $L_{3} \cap T_{3}$ are not empty is higher than $99.96 \%$ (see proof in Appendix C). Finally, we compute the values in $x_{3}$ and in the diagonal of $x_{4}$. 


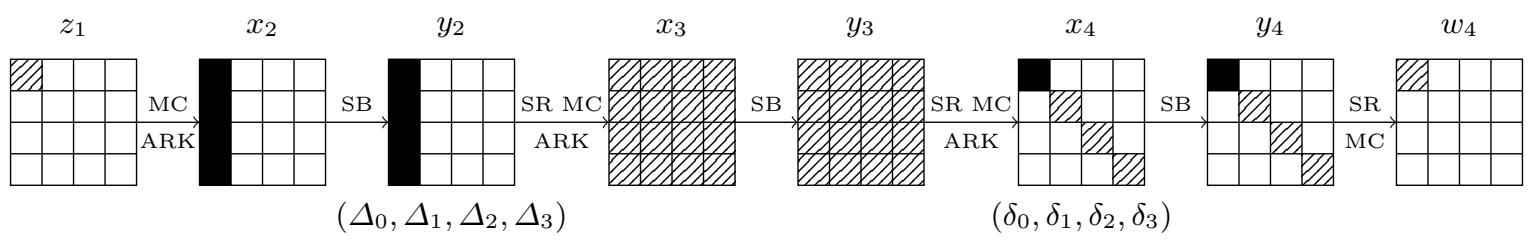

Figure 7: The 7-round distinguishing attack focuses of the middle rounds. Black bytes have known values and differences, gray bytes have known values, hatched bytes have known differences and white bytes have unknown values and/or differences.

We now need to find a key that matches the previous solving in the internal states: we build a partial pair of internal states that conforms to the middle rounds, but that sets 8 bytes on constraints in the key. Namely, if we denote $k_{i}$ the subkey introduced in round $i$ and $u_{i}=\mathrm{MC}^{-1}\left(k_{i}\right)$, then both $u_{3}$ and $k_{4}$ have four known bytes (see Figure 8). We start by

\begin{tabular}{|c|c|c|c|c|c|c|c|c|c|c|c|c|c|}
\hline \multicolumn{4}{|c|}{$u_{3}$} & & \multicolumn{4}{|c|}{$k_{3}$} & & \multicolumn{4}{|c|}{$k_{4}$} \\
\hline & 7 & 1 & 1 & & 3 & 7 & 2 & 2 & & & 8 & 8 & 8 \\
\hline 1 & 7 & 1 & & $\mathrm{MC}$ & 4 & 6 & 2 & 2 & KS & 5 & & 3 & 3 \\
\hline 1 & 7 & & 1 & & 4 & 6 & 2 & 2 & & 5 & 3 & & 3 \\
\hline 4 & & 1 & 1 & & 4 & 6 & 2 & 2 & & 5 & 3 & 3 & \\
\hline
\end{tabular}

Figure 8: Generating a compatible key: gray bytes are known, and numbers indicate the order in which we guess or determine the bytes.

fixing all the bytes marked by 1 in $u_{3}$ to random values: this allows to compute the values of all 2's in the two last columns of $k_{3}$. By the column-wise operations of AES key schedule, we can get the values of all bytes marked by 3 . As for the 4's, we get them since there are four known bytes among the eight in the first columns of $u_{3}$ and $k_{3}$. Again, the key schedule gives the 5's and 6's, and the MixColumns the 7's. Finally, we determine values for all the byte tagged by 8 from the key schedule equations. By inverting the key schedule, we are thus able to compute the master key $k$.

All in all, we start by getting a partial pair of internal states that conforms to the middle rounds, continue by deriving a valid key that matches the partial known bytes and determine the rest of the middle internal states to get the pair on input messages. The bottleneck of the time and memory complexity occurs when handling the lists of size at most $2^{8}$ elements to compute intersections. Note that those intersections can be done in roughly $2^{8}$ operations by representing lists by 256-bit numbers and then perform a logical AND.

In the end, we build a pair of messages $\left(m, m^{\prime}\right)$ and a key $k$ that conforms to the truncated differential characteristic of Figure 4 in time $2^{8}$ basic operations, where it costs $2^{64}$ in the generic scenario. We note that among the 18 freedom degrees left for the attack, we used only 10 by setting 10 bytes to random values, such that we expect $2^{8 \times 8}=2^{64}$ solutions in total. All those solutions could be generated in time $2^{64}$ by iterating over all the possibilities of the bytes marked by 1 in Figure 8 .

We implemented the described algorithm to verify that it indeed works, and we found for instance the triplet $\left(m, m^{\prime}, k\right)$ reported in Appendix A. 


\subsection{Distinguisher for 8-round AES-128}

We consider the 8-round truncated differential characteristic of Figure 9, where the matrices of differences in both the plaintext and the ciphertext lie in the same matrix subspaces of dimension four as before. Indeed, the output difference lies in a subspace of dimension four since all the operations after the last SubBytes layer are linear. Again, the distinguisher previously described (Section 3.1) claims that the time complexity to find a pair of messages that conforms to those patterns in a family of pseudo-random permutations runs in time $2^{64}$ operations.

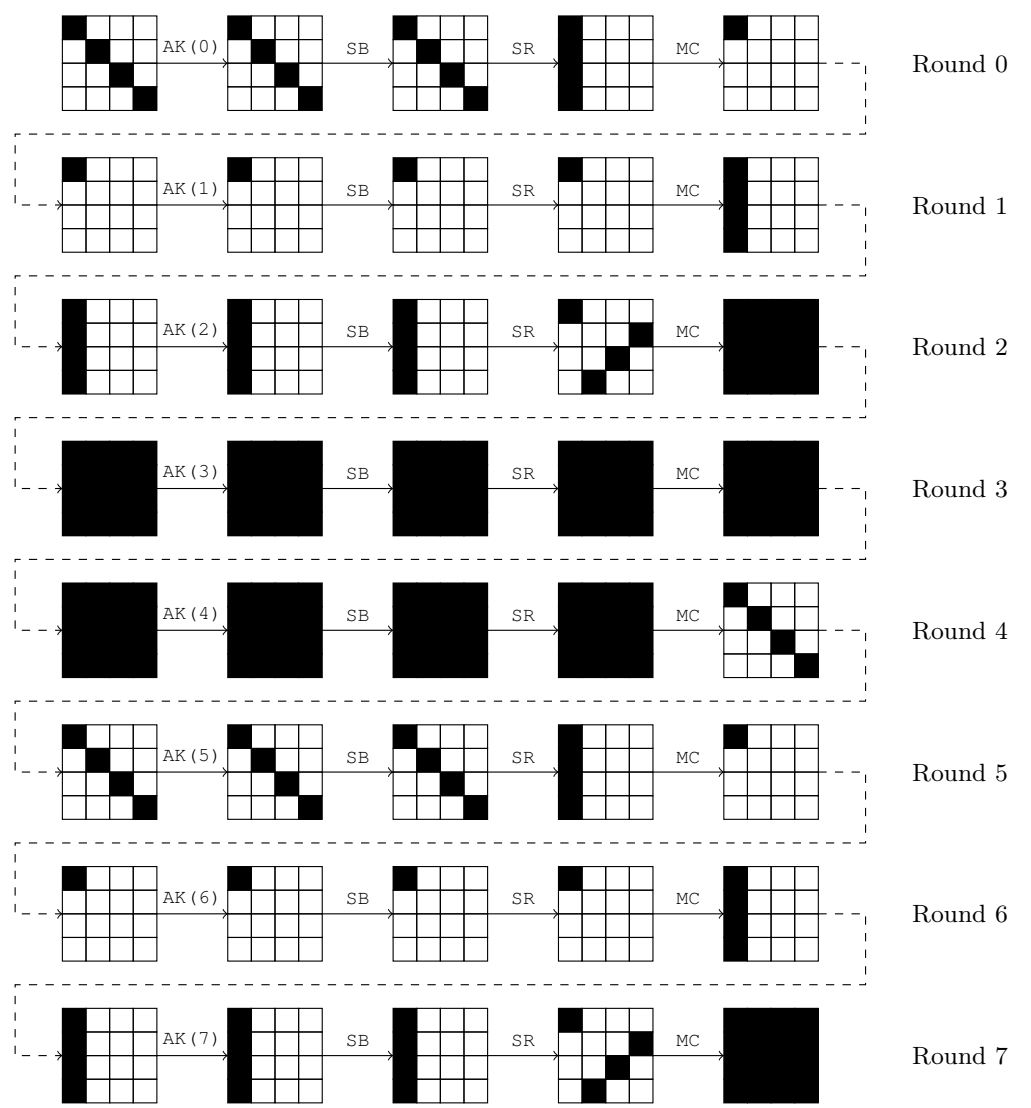

Figure 9: The 8-round truncated differential characteristic used to distinguish the AES-128. Black bytes are active, white bytes are not.

The following of this section describes a way to build a key and a pair of messages that conform to the restrictions in time and memory complexity $2^{24}$. We note that it is possible to optimize the memory requirement to $2^{16}$. As in the previous section, there are 36 freedom degrees at the input, which shrink to 18 after the consideration of the truncated differential characteristic. Therefore, we also expect $2^{8 \times 18}$ solutions in the end.

First of all, we observe that finding $2^{24}$ triplets $\left(m, m^{\prime}, k\right)$ composed by a key and a pair of internal states that conform to the rounds 2 to 5 is sufficient since the propagation in the outward rounds is done with probability $2^{-24}$ due to the MixColumns transition of round 1. The following analysis consequently focuses of those four middle rounds.

We now describe an instance of a problem that we use as a building block in our algorithm, which is related to the keyed SuperSBox construction. 
Problem 1. Let $a$ and $b$ two bytes. Given a 32-bit input and output differences $\Delta_{\text {in }}$ and $\Delta_{\text {out }}$ of a SuperSBox $_{k}$ for a unknown $k$, find all the pairs of AES-columns $\left(c, c^{\prime}\right)$ and keys $k$ such that:

$$
\begin{aligned}
\text { i. } & c+c^{\prime}=\Delta_{i n}, \\
\text { ii. } & \operatorname{SuperSBox}_{k}(c)+\operatorname{SuperSBox}_{k}\left(c^{\prime}\right)=\Delta_{\text {out }}, \\
\text { iii. } & \operatorname{SuperSBox}_{k}(c)=[a, b, \star, \star]^{\mathrm{T}} .
\end{aligned}
$$

Considering the key $k$ known and the case where there is no restriction on the output bytes (iii), we would expect this problem to have one solution on average. Finding it would naively require $2^{32}$ computations by iterating over the $2^{32}$ possible inputs and check whether the output has the correct $\Delta_{\text {out }}$ known difference. The additional constraints on the two output bytes reduce the success of finding a pair $\left(c, c^{\prime}\right)$ of input to $2^{-16}$, but if we allow the four bytes in the key $k$ to be chosen, then we expect $2^{16}$ solutions to this problem.

To find all of them in $2^{16}$ simple operations, we proceed as follows (Figure 10): the two output bytes $a$ and $b$ being known, we can deduce the values of the two associated bytes before the last SubBytes, $\tilde{a}$ and $\tilde{b}$ respectively. We can also deduce the difference in those bytes since the output difference is known. Then, we guess the two unset differences at the input of the last SubBytes: the differences then propagate completely inside the SuperSBox. At both SubBytes layers, by the differential properties of the AES S-Box, we expect to find one value on average for each of the six unset transitions. Consequently, the input and output of the AddRoundKey operation are known, which determines the four bytes of $k$. In the end, we find the $2^{16}$ solutions of Problem 1 in time $2^{16}$ operations.

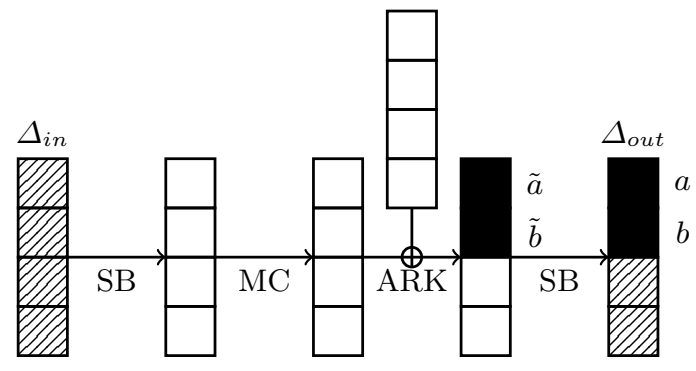

Figure 10: Black bytes have known values and differences, hatched bytes have known differences and white bytes have unknown values and/or differences.

To apply this strategy to the 8-round truncated differential characteristic of Figure 9, we start by randomizing the difference $\Delta y_{2}$, the difference $\Delta w_{5}$ and the values in the first column of $w_{5}$. Due to the linear operations involved, we deduce $\Delta x_{3}=\Delta w_{2}$ from $\Delta y_{2}$ and $\Delta y_{4}$ from $\Delta w_{4}$. To use the previous algorithm, we randomize the values of the two first columns of $w_{4}$ (situtation in Figure 11). Doing so, the four columns of $y_{4}$ are constrained on two bytes each and have fixed differences. Consequently, the four SuperSBoxes between $x_{3}$ and $y_{4}$ keyed by the four corresponding columns of $k_{4}$ conforms to the requirements ${ }^{3}$ of Problem 1 . In time and memory complexity $2^{16}$, for $i \in\{0,1,2,3\}$, we store the $2^{16}$ solutions for the $i$ th SuperSBox associated to the $i$ th column of $x_{4}$ in the list $L_{i}$.

We continue by observing that the randomization of the bytes in $w_{4}$ actually sets the value of two diagonal bytes in $k_{5}, k_{5}[0]$ and $k_{5}[5]$, which imposes constraints of the elements in the lists $L_{i}$. We start by considering the $2^{16}$ elements of $L_{3}$, and for each of them, we

\footnotetext{
${ }^{3}$ The positions of the known output bytes differ, but the strategy applies in the same way.
} 


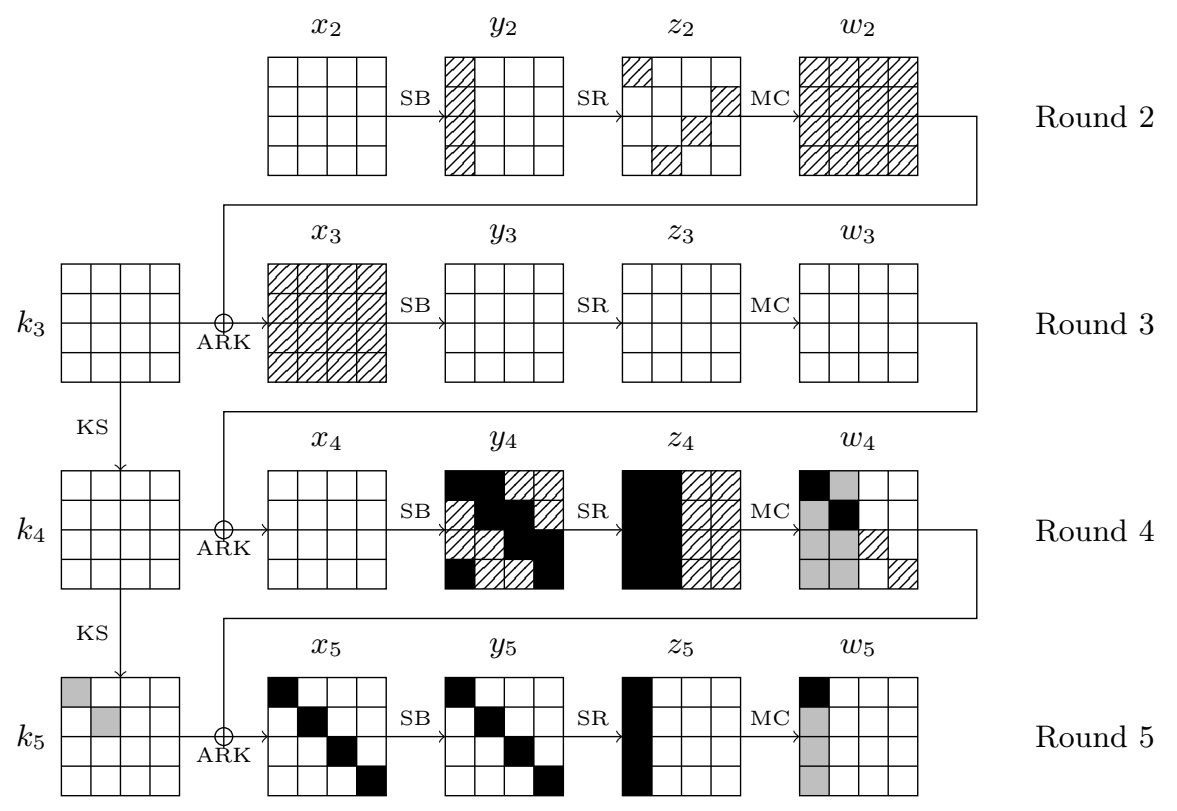

Figure 11: Black bytes have known values and differences, gray bytes have known values, hatched bytes have known differences and white bytes have unknown values and/or differences.

learn the values $x_{4}[12 . .15]$ and $k_{4}[12 . .15]$. Due to the column-wise operations in the key schedule, we also deduce the value of $k_{4}[0]$. Filtering the elements of $L_{0}$ which share that value of $k_{4}[0]$, we are left with $2^{8}$ elements for bytes $x_{4}[0 . .3]$ and $k_{4}[0 . .3]$. At this point, we constructed $2^{16+8}=2^{24}$ solutions in time $2^{24}$ that we store in a list $L_{0,3}$.

As $k_{5}[5]$ has been previously determined, we can deduce $k_{4}[5]=k_{5}[5]+k_{5}[1]$ from the AES key schedule for each of the entry of $L_{0,3}$. Again, this adds an 8-bit constraint on the elements of $L_{1}$ : we expect $2^{8}$ of them to match the condition on $k_{4}[5]$. In total, we could construct a list $L_{0,1,3}$ of size $2^{24+8}=2^{32}$, whose elements would be the columns 0,1 and 3 of $x_{4}$ and $k_{4}$, but as soon as we get $2^{24}$ elements in that list, we stop and discard the remaining possibilities.

Finally, to ensure the correctness of the choice in the remaining column 2, we need to consider the MixColumns operation in round 4 and the subkey $k_{5}$. Indeed, as soon as we choose an element in both $L_{0,1,3}$ and $L_{2}, x_{4}, k_{4}$ and $k_{5}$ become fully determined, but we need to ensure that the values $x_{5}[10]$ and $x_{5}[15]$ equal to the known ones. In particular, for $x_{5}[10]$, we have:

$$
\begin{aligned}
k_{4}[10]+k_{5}[6] & =k_{5}[10] \\
& =w_{4}[10]+x_{5}[10] \\
& =z_{4}[8]+z_{4}[9]+2 z_{4}[10]+3 z_{4}[11]+x_{5}[10],
\end{aligned}
$$

and for $x_{5}[15]$ :

$$
\begin{aligned}
k_{4}[11]+k_{5}[7]+k_{4}[15] & =k_{5}[11]+k_{4}[15] \\
& =k_{5}[15] \\
& =w_{4}[15]+x_{5}[15] \\
& =3 z_{4}[12]+z_{4}[13]+z_{4}[14]+2 z_{4}[15]+x_{5}[15],
\end{aligned}
$$

where (3), (6) and (7) come from the key schedule, (4) and (8) from the AddRoundKey and (5) and (9) use the equations from the MixColumns. Hence, for each element of $L_{0,1,3}$, 
we can compute:

$$
\begin{aligned}
\mathrm{S}\left(x_{4}[8]\right)+k_{4}[10] & :=x_{5}[10]+k_{5}[6]+\mathrm{S}\left(x_{4}[13]\right)+2 \mathrm{~S}\left(x_{4}[2]\right)+3 \mathrm{~S}\left(x_{4}[7]\right), \\
k_{4}[11]+2 \mathrm{~S}\left(x_{4}[11]\right) & :=k_{5}[7]+k_{4}[15]+3 \mathrm{~S}\left(x_{4}[12]\right)+\mathrm{S}\left(x_{4}[1]\right)+\mathrm{S}\left(x_{4}[6]\right)+x_{5}[15]
\end{aligned}
$$

and lookup in $L_{2}$ to find $2^{16} 2^{-8 \times 2}=1$ element that match those two byte conditions. We create the list $L$ by adding the found element from $L_{2}$ to each entry of $L_{0,1,3}$.

All in all, in time and memory complexity $2^{24}$, we build $L$ of size $2^{24}$ and we now exhaust its elements to find one that passes the $2^{-24}$ probability of the $4 \rightarrow 1$ backward transition in the MixColumns of round 1. Indeed, an $a \rightarrow b$ transition in the MixColumns layer cancels $4-b$ output bytes, so that it would happen with probability $2^{-8(4-b)}$ for a random input $a$. Consequently, we expect to find a pair $\left(m, m^{\prime}\right)$ of messages and a key $k$ that conforms to the 8 -round truncated differential characteristic of Figure 9 in time $2^{24}$ when it requires $2^{64}$ computations in the ideal case.

Among the 18 available freedom degrees available to mount the attack, we uses 17 of them, which means that we expect to have $2^{8}$ solutions. We could have them in time $2^{32}$, but since we discarded $2^{8}$ elements in the algorithm described, we get only 1 in time $2^{24}$. We note that it is possible to gain a factor $2^{8}$ in the memory requirements of our attack since we can implement the algorithm without storing the lists $L_{0}, L_{0,3}$ and $L_{0,1,3}$, by using hash tables for $L_{1}, L_{2}$ and $L_{3}$.

We also implemented the described algorithm to verify that it indeed works, and we found for instance the triplet $\left(m, m^{\prime}, k\right)$ reported in Appendix B.

\section{Extention to AES-256}

The two distinguishers described in the previous section can be easily extended in distinguishers on the AES-256. The main idea is to use the 16 additional freedom degrees in the key to extend the truncated differential characteristics by introducing a new fully active round in the middle.

\subsection{Distinguisher for 7-round $A E S-256$}

The first step of the attack described in the 7-round distinguisher on AES-128 (Section 3.2) still applies in the case of AES-256 since it does not involve the key schedule. Then, we can generate a compatible key easily since there are only two subkeys involved: we can just choose bytes of $k_{3}$ and $k_{4}$ as we want, except the imposed ones, and deduce the master key afterwards. This yields to a distinguisher with time and memory complexities around $2^{8}$.

\subsection{Distinguisher for 8-round $A E S-256$}

We use a similar approach as the 7-round distinguisher on AES-128 of Section 3.2, but the truncated differential characteristic has one more fully active round in the middle ${ }^{4}$.

We begin by choosing values for $\Delta z_{1}$ and $x_{2}[0 . .3]$. This allows to deduce $\Delta x_{2}, \Delta y_{2}$, and $\Delta x_{3}$. Then, we also set random values for $\Delta w_{5}$ and for the diagonal of $x_{5}$ to obtain both $\Delta x_{5}$ and $\Delta y_{4}$. Now, we find a value for $\Delta x_{4}$, which is compatible with $\Delta x_{3}$ and $\Delta y_{4}$. Indeed, we can not take an arbitrary value for $\Delta x_{4}$ because the probability that it fits is very close to $2^{-32}$. However, we can find a correct value with the following steps:

\footnotetext{
${ }^{4}$ In that case, the truncated differential characteristic is thus the one from Figure 9.
} 
1. Store the $2^{7}$ possible values for $\Delta x_{4}[0]$ in a list $L_{0}$.

2. In a similar way, make lists $L_{1}$ with $\Delta x_{4}[1], L_{2}$ with $\Delta x_{4}[2]$ and $L_{3}$ with $\Delta x_{4}[3]$.

3. Choose a value for $\left(x_{3}[0], x_{3}[5], x_{3}[10], x_{3}[15]\right)$ and compute $\Delta x_{4}[0 . .3]$.

4. If $\Delta x_{4}[0 . .3]$ is not in $L_{0} \times L_{1} \times L_{2} \times L_{3}$, then go back to step 3 .

On average, we go back to the step 3 only $\left(2^{8-7}\right)^{4}=2^{4}$ times since lists are of size $2^{7}$. In the same way, we can obtain values for the other columns of $x_{4}$.

At this point, we computed actual values in all those internal states, and we need to generate a compatible key. Finding one can be done using the procedure described in Figure Figure 12. Bytes tagged by 1 are chosen at random, odd steps use the key schedule equations and even steps the properties of MixColumns.

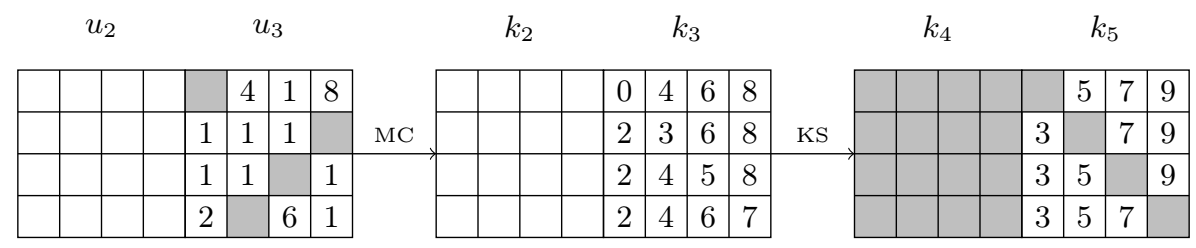

Figure 12: Generating a compatible key: gray bytes are known, and numbers indicate the order in which we guess or determine the bytes.

\subsection{Distinguisher for 9-round AES-256}

We begin as in Section 3.3 by choosing the difference $\Delta y_{2}$, the difference $\Delta w_{6}$ and the values in the first column of $w_{6}$. Then, we deduce $\Delta w_{2}=\Delta x_{3}$ from $\Delta y_{2}$ and $\Delta y_{5}$ from $\Delta w_{5}$. In addition, we set $x_{3}$ to a random value, which allows to determine $\Delta x_{4}$. In order to apply the result from Problem 1 again, we set the values in two first columns of $w_{5}$ to random values.

As before, for $i \in\{0,1,2,3\}$, we store in the list $L_{i}$ the $2^{16}$ possible values of the $i$-th column of $x_{5}$ and the $i$-th column of $k_{5}$. Unlike previously, we also obtain values of the $i$-th column of $\mathrm{SR}\left(k_{4}\right)$, but the scenario of the attack still applies. We start by observing that bytes of $L_{0}$ allow to compute $k_{4}[1]$ and $k_{4}$ [13], which are bytes of $L_{3}$. Thus, we can merge $L_{0}$ and $L_{3}$ in a list $L_{0,3}$ containing $2^{16}$ elements. Then, we construct the list $L_{0,2,3}$ containing $2^{24}$ elements of $L_{0,3} \times L_{2}$. Finally, from bytes of $L_{0,2,3}$, we can compute:

$$
\begin{aligned}
3 z_{5}[11] & :=k_{4}[2]+\mathrm{S}\left(k_{5}[15]\right)+k_{4}[6]+k_{4}[10]+z_{5}[8]+z_{5}[9]+2 z_{5}[10]+x_{6}[10], \quad(12) \\
z_{5}[14]+k_{4}[3] & :=\mathrm{S}\left(k_{5}[12]\right)+k_{4}[7]+k_{4}[11]+k_{4}[15]+3 z_{5}[12]+z_{5}[13]+2 z_{5}[15]+x_{6}[15] .
\end{aligned}
$$

As a consequence, we expect only one element of $L_{1}$ to satisfy those two byte conditions and so, we obtain $2^{24}$ solutions for the middle rounds. All in all, this yields to a distinguisher with a time complexity around $2^{24}$ and a memory requirement around $2^{16}$ using the same trick given in Section 3.3.

\section{Conclusion}

In this paper, we study the Advanced Encryption Standard and show how to find a pair of messages and a key that satisfy some property a lot more efficiently than a generic attack based on the birthday paradox for both AES-128 and AES-256. Our new results improve 
the previous claimed ones by reaching very practical complexities, and give new insights of the open-key model for block ciphers, and hash functions based on block ciphers.

On AES-128, we show efficient distinguishers for versions reduced to seven and eight rounds, and verified in practice that they indeed work by implementing the actual attacks. We describe precisely the algorithms to get the valid inputs, and by applying the same strategy, we deduce similar results for AES-256. Namely, we get efficient distinguishers on versions reduced to seven, eight and nine rounds.

\section{References}

1. Biryukov, A., Khovratovich, D.: Related-Key Cryptanalysis of the Full AES-192 and AES-256. [11] $1-18$

2. Biryukov, A., Khovratovich, D., Nikolic, I.: Distinguisher and Related-Key Attack on the Full AES-256. In Halevi, S., ed.: CRYPTO. Volume 5677 of Lecture Notes in Computer Science., Springer (2009) 231249

3. Biryukov, A., Nikolic, I.: A New Security Analysis of AES-128. CRYPTO 2009 rump session, slides only (2009)

4. Bogdanov, A., Khovratovich, D., Rechberger, C.: Biclique Cryptanalysis of the Full AES. In Lee, D.H., Wang, X., eds.: ASIACRYPT. Volume 7073 of Lecture Notes in Computer Science., Springer (2011) 344-371

5. Bouillaguet, C., Derbez, P., Fouque, P.A.: Automatic search of attacks on round-reduced AES and applications. In Rogaway, P., ed.: CRYPTO. Volume 6841 of Lecture Notes in Computer Science., Springer (2011) 169-187

6. Daemen, J., Rijmen, V.: Understanding Two-Round Differentials in AEs. In Prisco, R.D., Yung, M., eds.: SCN. Volume 4116 of Lecture Notes in Computer Science., Springer (2006) 78-94

7. Dunkelman, O., Keller, N., Shamir, A.: Improved Single-Key Attacks on 8-Round AES-192 and AES-256. In Abe, M., ed.: ASIACRYPT. Volume 6477 of Lecture Notes in Computer Science., Springer (2010) $158-176$

8. Gilbert, H., Peyrin, T.: Super-Sbox Cryptanalysis: Improved Attacks for AES-Like Permutations. In Hong, S., Iwata, T., eds.: FSE. Volume 6147 of Lecture Notes in Computer Science., Springer (2010) 365-383

9. Knudsen, L.R., Rijmen, V.: Known-Key Distinguishers for Some Block Ciphers. In Kurosawa, K., ed.: ASIACRYPT. Volume 4833 of Lecture Notes in Computer Science., Springer (2007) 315-324

10. Lamberger, M., Mendel, F., Rechberger, C., Rijmen, V., Schläffer, M.: Rebound Distinguishers: Results on the Full Whirlpool Compression Function. [11] 126-143

11. Matsui, M., ed.: Advances in Cryptology - ASIACRYPT 2009, 15th International Conference on the Theory and Application of Cryptology and Information Security, Tokyo, Japan, December 6-10, 2009. Proceedings. In Matsui, M., ed.: ASIACRYPT. Volume 5912 of Lecture Notes in Computer Science., Springer (2009)

12. Mendel, F., Peyrin, T., Rechberger, C., Schläffer, M.: Improved Cryptanalysis of the Reduced Grøst 1 Compression Function, ECHO Permutation and AES Block Cipher. In Jr., M.J.J., Rijmen, V., SafaviNaini, R., eds.: Selected Areas in Cryptography. Volume 5867 of Lecture Notes in Computer Science., Springer (2009) 16-35

13. Mendel, F., Rechberger, C., Schläffer, M., Thomsen, S.S.: The Rebound Attack: Cryptanalysis of Reduced Whirlpool and Grøstl. In Dunkelman, O., ed.: FSE. Volume 5665 of Lecture Notes in Computer Science., Springer (2009) 260-276

14. Minier, M., Phan, R.C.W., Pousse, B.: Distinguishers for Ciphers and Known Key Attack against Rijndael with Large Blocks. In Preneel, B., ed.: AFRICACRYPT. Volume 5580 of Lecture Notes in Computer Science., Springer (2009) 60-76

15. Nikolic, I., Pieprzyk, J., Sokolowski, P., Steinfeld, R.: Known and Chosen Key Differential Distinguishers for Block Ciphers. In Rhee, K.H., Nyang, D., eds.: ICISC. Volume 6829 of Lecture Notes in Computer Science., Springer (2010) 29-48

16. NIST: Advanced Encryption Standard (AES), FIPS 197. Technical report, NIST (November 2001)

17. Sasaki, Y., Emami, S., Hong, D., Kumar, A.: Improved known-key distinguishers on feistel-sp ciphers and application to camellia. In Susilo, W., Mu, Y., Seberry, J., eds.: ACISP. Volume 7372 of Lecture Notes in Computer Science., Springer (2012) 87-100

18. Sasaki, Y., Yasuda, K.: Known-Key Distinguishers on 11-Round Feistel and Collision Attacks on Its Hashing Modes. In Joux, A., ed.: FSE. Volume 6733 of Lecture Notes in Computer Science., Springer (2011) 397-415 


\section{A Solution for the 7-round truncated differential characteristic on AES-128}

Table 2: Example of a pair of messages $\left(m, m^{\prime}\right)$ that conforms to the 7 -rounds truncated differential characteristic for AES-128 of Section 3.2. The master key found by the attack is: 93CA1344 10A7EBDF B659C8AF ECC59699. The lines in this array contains the values of two internal states before entering the corresponding round, as well as their difference.

\begin{tabular}{|c|c|c|c|}
\hline Round & $m$ & $m^{\prime}$ & $m \oplus m^{\prime}$ \\
\hline Init. & E5FC5DFE 79A851F7 7EB9E366 51C3D9C5 & F8FC5DFE 79C951F7 7EB96566 51C3D96E & $1 D 0000000061000000008$ \\
\hline 0 & $76364 \mathrm{EBA} 690 \mathrm{FBA} 28 \mathrm{C} 8 \mathrm{E} 02 \mathrm{BC} 9 \mathrm{BD} 064 \mathrm{~F} 5 \mathrm{C}$ & 6B364EBA 696EBA28 C8E0ADC9 BD0 & 1D0000000061000000 \\
\hline 1 & CC94D1 85BE1AD 3 F3D75BF1 ACCBB8BD & 8DCC94D1 85BE1AD 3 F 3L & $E 8000000000000000000000000$ \\
\hline 2 & E93319CD 88F41390 $10623230 \mathrm{~F} 66 \mathrm{BFBAD}$ & C92309FD 88F41390 10 & 2010103000000000000 \\
\hline 3 & 89C79074 E09E6F44 F1DBAB2F F984FCC4 & $1404532 \mathrm{~A} 09774 \mathrm{~F} 8 \mathrm{D}$ 24BF1AFA CD551921 & 9DC3C35E E9E920C9 D5 \\
\hline 5 & 84606BEA OE22D904 3BF29061 9F454807 & 4B606BEA 0E22D904 3BF29061 9F454807 & CF 000000000000000000000000000000 \\
\hline 6 & FF867544 274436AF 75ECC287 A6BF72F6 & 3C6A996B 274436AF 75ECC287 A6BF72F 6 & C3ECEC2F 000000000000000000000000 \\
\hline End & C49E4CB3 0C944043 D5ED6D3B 247E3843 & 2563B1AF 68F0EC8B A6788B48 EEF27E05 & E1FDFD1C 6464 ACC 8 7395E 673 CA8C4646 \\
\hline
\end{tabular}

\section{B Solution for the 8-round truncated differential characteristic on AES-128}

Table 3: Example of a pair of messages $\left(m, m^{\prime}\right)$ that conforms to the 8-round truncated differential characteristic for AES-128 of Section 3.3. The master key found by the attack is: 98C45623 6CA00686 301E836D 614DFAB0. The lines in this array contains the values of two internal states before entering the corresponding round, as well as their difference.

\begin{tabular}{|c|c|c|c|}
\hline Round & $m$ & $m^{\prime}$ & $m \oplus m^{\prime}$ \\
\hline Init. & 9588B342 D43D04D 4 AB298AE1 E43687DB & OB88B342 D46904D4 AB29D0E1 E4368728 & 9E0000000054000000005A00000 \\
\hline 0 & 61 B89D02529B & $934 \mathrm{CE} 561$ B8C90252 9B3 & 9E000000 0054000000005A0000 \\
\hline 1 & 53FEBB0F 6BFF8E5E B & OEFEBB0F 6BFF8E5E B471A8E3 1A2232A3 & 5D0000000000000000 \\
\hline 2 & E9F44380 991A8ECB F7B18344 2C936CEB & 65B2054A 991A8ECB F7B18344 2C936CEB & $8 \mathrm{C} 4646 \mathrm{CA} 000000000000000000$ \\
\hline 3 & 2977F 65C 3883EDEF 615D3C9E 5CE5384B & 8F24A5A9 2398COD9 10CEDEEF DFEEBOC3 & A65353F5 1B1B2D36 7193E271 83 \\
\hline 5 & C3E1961D 02A9713E 770A20D4 547 & 8DE1961D 029B713E 770A3AD4 5470FA27 & $4 \mathrm{E} 0000000032000000001 \mathrm{~A} 0000$ \\
\hline 6 & D79D534C 33CC3861 76635DCD 548870C9 & EB9D534C 33CC3861 76635DCD 548870C9 & $3 C 0000000000000000$ \\
\hline 7 & D7F645C6 89358035 09847940 D831EFDE & 0211A2F4 8935803509847940 D831EFDE & D5E7E732000000000000000000000000 \\
\hline End & 16E58308 DFD78F11 A8B05B9D C0A0363E & E49CFA83 D 4DC9207 FC4CF3C9 9B3BF 6FE & F279798B 0B0B1D16 54FCA854 5B9H \\
\hline
\end{tabular}




\section{Probability of success}

We are interested in the probability that the intersection of four or five subsets of $\{1, \ldots, 255\}$ each of size 128 being empty.

To evaluate it, let $\mathcal{P}$ denote the set of subsets $X \subset\{1, \ldots, 255\}$ such that $|X|=128$. We also define:

$$
T(n, k):=\left\{\left(X_{1}, \ldots, X_{n}\right) \in \mathcal{P}^{n}|| X_{1} \cap \ldots \cap X_{n} \mid=k\right\} \quad \text { for } n \geq 1, k \geq 0 .
$$

In others words, $|T(n, k)| /\left|\mathcal{P}^{n}\right|$ is the probability that the intersection of $n$ elements from $\mathcal{P}$ has a size equal to $k$.

Property 2 The cardinality of $T(n, k)$ satisfies the following recurrence relation:

$$
\left\{\begin{aligned}
|T(1, k)| & =|\mathcal{P}| \text { if } k=128,0 \text { otherwise } \\
|T(n+1, k)| & =\sum_{l=k}^{128}|T(n, l)|\left(\begin{array}{l}
l \\
k
\end{array}\right)\left(\begin{array}{l}
255-l \\
128-k
\end{array}\right) \quad \text { for } n \geq 1, k \geq 0 .
\end{aligned}\right.
$$

Proof. First, we note that we can partition $\mathcal{P}^{n}$ by the sets:

$T(n, Y):=\left\{\left(X_{1}, \ldots, X_{n}\right) \in \mathcal{P}^{n} \mid X_{1} \cap \ldots \cap X_{n}=Y\right\} \quad$ for any subset $Y \subset\{1, \ldots, 255\}$.

Then, we have:

$$
\begin{aligned}
|T(n+1, k)| & =\sum_{Y}\left|\left\{\left(X_{1}, \ldots, X_{n+1}\right) \in T(n, Y) \times \mathcal{P}|| Y \cap X_{n+1} \mid=k\right\}\right| \\
& =\sum_{Y}|T(n, Y)| \times|\{X \in \mathcal{P}|| Y \cap X \mid=k\}|
\end{aligned}
$$

If we fix a set $Y \subset\{1, \ldots, 255\}$, then a set $X \in \mathcal{P}$ such that $|X \cap Y|=k$ is obtained by choosing $k$ elements in $Y$ and $128-k$ elements in $Y^{c}$. As a consequence, we obtain:

$$
\begin{aligned}
|T(n+1, k)| & =\sum_{Y}|T(n, Y)|\left(\begin{array}{c}
|Y| \\
k
\end{array}\right)\left(\begin{array}{c}
255-|Y| \\
128-k
\end{array}\right) \\
& =\sum_{l=0}^{255}\left(\begin{array}{l}
l \\
k
\end{array}\right)\left(\begin{array}{c}
255-l \\
128-k
\end{array}\right) \sum_{|Y|=l}|T(n, Y)|
\end{aligned}
$$

Finally, we remark that $\{T(n, Y)\}_{|Y|=l}$ is a partition of $T(n, l)$ and thus:

$$
|T(n+1, k)|=\sum_{l=0}^{255}\left(\begin{array}{l}
l \\
k
\end{array}\right)\left(\begin{array}{c}
255-l \\
128-k
\end{array}\right)|T(n, l)| .
$$

Using Maple, we found that the probability of failure of the distinguisher described in Section 3.2 is:

$$
\frac{T(4,0)}{|\mathcal{P}|^{4}} \times\left(\frac{T(5,0)}{|\mathcal{P}|^{5}}\right)^{3} \approx 0.04 \%
$$

